\title{
Collapsing granular suspensions
}

\section{Journal Article}

\section{Author(s):}

Kadau, Dirk; Andrade Jr., José S.; Herrmann, Hans Jürgen

Publication date:

2009-11

Permanent link:

https://doi.org/10.3929/ethz-b-000016227

Rights / license:

In Copyright - Non-Commercial Use Permitted

Originally published in:

The European Physical Journal E 30(3), https://doi.org/10.1140/epje/i2009-10523-6 


\title{
Collapsing granular suspensions
}

\author{
D. $\operatorname{Kadau}^{1, \mathrm{a}}$, J.S. Andrade Jr. ${ }^{1,2}$, and H.J. Herrmann ${ }^{1,2}$ \\ 1 IfB, HIF E12, ETH Hönggerberg, 8093 Zürich, Switzerland \\ 2 Departamento de Física, Universidade Federal do Ceará, 60451-970 Fortaleza, Ceará, Brazil
}

Received 13 October 2008 and Received in final form 24 August 2009

Published online: 11 October 2009 - (c) EDP Sciences / Società Italiana di Fisica / Springer-Verlag 2009

\begin{abstract}
A 2D contact dynamics model is proposed as a microscopic description of a collapsing suspension/soil to capture the essential physical processes underlying the dynamics of generation and collapse of the system. Our physical model is compared with real data obtained from in situ measurements performed with a natural collapsing/suspension soil. We show that the shear strength behavior of our collapsing suspension/soil model is very similar to the behavior of this collapsing suspension soil, for both the unperturbed and the perturbed phases of the material.
\end{abstract}

PACS. 47.57.-s Complex fluids and colloidal systems - 45.70.Mg Granular flow: mixing, segregation and stratification - 83.80.Hj Suspensions, dispersions, pastes, slurries, colloids

\section{Introduction}

In nature, fragile or metastable granular structures, collapsing under an applied load exist in a large variety. For instance, these collapsing suspensions/soils are well known from soil mechanics as an unsaturated (by water) grain structure which collapses when adding water (thus becoming saturated) or/and when loaded. They are also known to be loose structures and can be water deposited or aeolian [1-3]. Similar structures are also responsible for collapsible behavior of colloidal gels [4] or even snow [5].

In the present work we develop and numerically solve a simple physical model for a collapsing suspension/soil representation, that is capable of incorporating at the microscopic level the essential structural and dynamical features of the material when subjected to different types of perturbations. Within this context we focus on two aspects of the problem, namely the penetration of an intruder, leading to a partial collapse of the system, and the shear resistance property of the material. The model results of our approach are then compared to real and in situ data of a collapsing suspension/soil.

\section{Simulation method}

The discrete element method constitutes a general class of modeling techniques to simulate the microscopic behavior (i.e., at the particle scale) of granular/soil materials. Here

\footnotetext{
a e-mail: dkadau@ethz.ch
}

a variant of the contact dynamics method, originally developed to model compact and dry systems with lasting contacts [6], is used to describe a $2 \mathrm{D}$ cohesive suspension/soil material. This technique is conceptually based on the exact implementation of non-smooth contact laws, which means that the steric volume exclusion for perfectly rigid particles and the Coulomb friction law are strictly implemented $[7,8]$.

The absence of cohesion between particles can only be justified in dry systems on scales where the cohesive force is weak compared to the gravitational force on the particle, i.e. for dry sand and coarser materials, which can lead to densities close to that of random dense packings. For collapsing suspensions/soils, an attractive force must play an important role in the stabilization of large voids $[9,10]$, leading to highly porous systems as, e.g., in fine cohesive powders. In the nanometer range of particle sizes, the cohesive force becomes the dominant force, so that particles stick together upon first contact. Here the bonding between two particles is considered in terms of a cohesion model with a constant attractive force $F_{c}$ acting within a finite range $d_{c}$, so that for the opening of a contact a finite energy barrier $F_{c} d_{c}$ must be overcome. The utilization of this simplified cohesion model is justified in the case of short-range interactions as we are not interested in the detailed behavior of an individual contact, but on the collective behavior of the system, where the opening of a contact is mostly determined by the force and energy barrier. More precisely, using a more complex model would not lead to a drastic change in the macroscopic behavior when keeping the same force and energy barrier. In most force-driven simulation as in this paper the cohesion 
force dominates the behavior and the energy barrier plays a minor role but its existence is of crucial importance to avoid simulation artefacts $[9,8]$. As we show later, this cohesion force can be mediated, for example, by bacteria present in the system which are much smaller than the grains, i.e. we have an effective short-range interaction. We use $d_{c}=10^{-4}$ (in units of particle radii) for all the following simulations. In addition, we implement friction and rolling friction between two particles in contact, so that large pores can be stable in the system. For comparison, we adopt the same friction coefficient $\mu=0.3$ and coefficient for rolling friction $\mu_{r}=0.1$ (in units of particle radii) in all simulations, taking into account that rolling of particle contacts is easier than sliding $[9,8]$.

In the case of collapsing suspensions/soils one also has to take into account the time necessary for bonds to appear, i.e. during relatively fast processes new bonds will not be formed, whereas for long-term processes bonds are allowed to form at a particle contact. Finally, gravity also cannot be neglected in the model since the particle diameter is usually well above the micron size. For simplicity, however, the surrounding pore water is not explicitly considered but only taken into account as a buoyant medium, reducing the effective gravity acting onto the grains. In this way, we disregard fluid motion. This hypothesis should remain valid for the settling grains in the system as long as the velocities reached by each particle are sufficiently large (i.e., inertial effects are relevant), and the total virtual buoyancy induced by all particles released during the penetration process is small [11]. The issue of disregarding the fluid motion will be discussed in more detail when presenting the results in the following section.

\section{Results}

As mentioned earlier the major feature of the metastable granular structures investigated in this paper is that they collapse, e.g. when an external load is applied. This will be illustrated and investigated in sect. 3.1 by penetration of an intruder leading to partial collapse of the structures. Understanding the penetration behavior will also be important for the experiments presented in sect. 3.3, where the measurement device has to be pushed inside the soil before the measurements. Before and after the collapse the shear strength of the material changes drastically as shown in the simulations (sect. 3.2) and in experiments (sect. 3.3).

A useful number for the characterization of the structures after the (partial) collapse where many bonds break is the fraction of still active bonds (i.e. fully cohesive) with respect to the total number of contacts. This number will be used in the following for interpretation of the results.

\subsection{Penetration of an intruder}

We carry out simulations of a large disk (intruder) of low density (half the grain density) pushed with constant force into the granular structure and subsequently
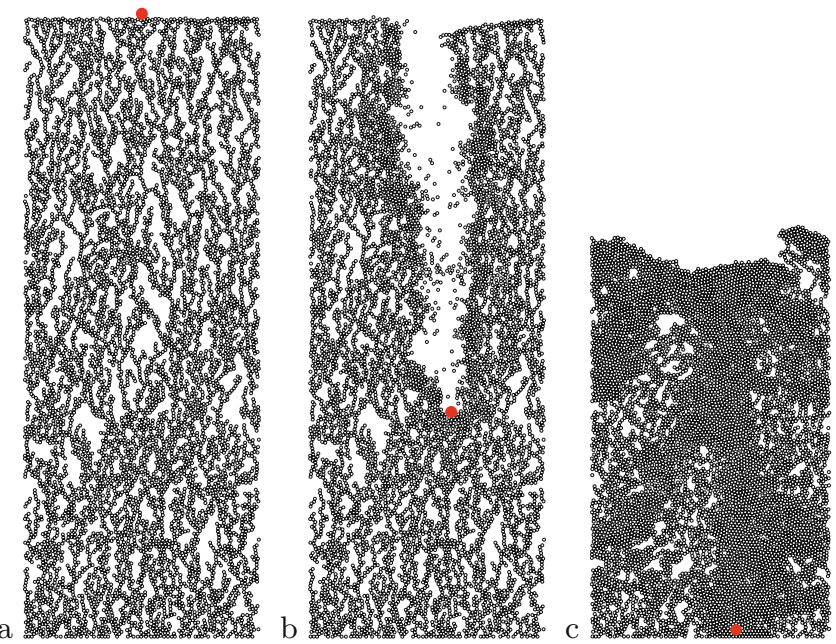

Fig. 1. Snapshots from simulations showing a typical realization of the penetration process of an intruder into a very loose cohesive packing. The (unperturbed) collapsing suspension/soil shown in (a) is modeled as a tenuous granular network of cohesive disks assembled by a gravity-driven process of ballistic deposition and contact dynamics [6,9]. The system size is $51 \times 199$ in units of grain diameters, corresponding to $1.7 \mathrm{~cm} \times 6.7 \mathrm{~cm}$ when using the typical diameter $d=344 \mu \mathrm{m}$ of the grains in the experiments. As shown in (b), the movement of the intruder is responsible for the partial destruction of the granular structure along its trajectory. At the end (c), the intruder rests under a compact mass of (perturbed) material.

removed again from a fragile cohesive granular structure. Only if the force exceeds a certain threshold it penetrates the medium. Additionally, we assume that the time scale for the packing generation is large enough to create bonds between the grains, whereas the movement of the intruder is on time scales much smaller than the bonding time. The sequence of snapshots shown in fig. 1 corresponds to different stages of the penetration process into a typical realization of a very loose packing. The initial (unperturbed) network structure (fig. 1a) is a result of the aggregation of cohesive disks by means of a gravity-driven process which includes ballistic deposition with contact dynamics $[6,9]$. At this point we are mostly interested in the penetration process into a fragile structure independent of its process of generation. The specific configurations used here are generated to resemble as closely as possible the experiments described later (sect. 3.3). The details of this generation process will be described later (sect. 3.2). The initial configurations used here (fig. 1) are created with a value $F_{c} / F_{g}=10^{4}$ for the cohesion force $F_{c}$ normalized by the gravitational force $F_{g}$ on each grain. Deliberately we chose this high value to obtain a rather loose structure with coordination number slightly above 2 (2.06), since lower values of $F_{c} / F_{g}$ lead to denser structures (cf. sect. 3.2) with smaller pores, i.e. a more homogeneous microstructure as found for non-cohesive materials and therefore relatively dense materials in laboratory experiments [12-14].

Note that the microstructure of two dimensional systems gives, at first glance, a less homogeneous impression 
than the corresponding three-dimensional systems, as pores are much easier to identify visually. This has been illustrated, e.g. for the compaction of cohesive powders when comparing two-dimensional simulations [9] to threedimensional simulations [15].

Once the intruder breaks through, the highly porous material collapses under the action of gravity. We observe the creation of a channel (fig. 1b) which finally collapses over the descending intruder. At the end, the partial destruction of the network due to the penetration process generates a large amount of compact (perturbed) material over the intruder. As shown in fig. 1c, the larger disk is buried under the debris of particles. In this case of a relatively "strong penetration" (cf. following paragraph) the fraction of active bonds for the total packing is about $30 \%$. This means that about $43 \%$ of the cohesive contacts in the initial configuration remain intact as during the collapse new contacts are formed, leading to an increase in the average coordination number to about 2.82. These numbers, averaged over the whole systems, can be seen as a measure for the extent of still intact areas and collapsed areas. In the fully intact areas still all bonds are active and the coordination number is unchanged, slightly above 2 . On the contrary, in the fully collapsed area, i.e. the channel created by the intruder, only about $2 \%$ of the bonds are still active, and the coordination number is about 3.2. In this respect the total numbers can also be viewed as an indicator for the collapsed fraction of the structure.

The results presented above clearly suggest that objects lighter than water can be effectively swallowed in a collapsing suspension/soil. Furthermore, if we assume that cohesive bonds can be restored by some particular physico-chemical or biological mechanism, the force needed to remove the intruder disk could be significantly higher than the originally penetration force. In order to provide a more quantitative confirmation for this interesting behavior, several simulations of the intruder penetration/removal process have been performed for 10 different realizations of granular networks generated with the same microscopic cohesion and friction parameters. In fig. 2 we show the dependence of the weight fraction $w / w_{T}$ on the ratio $F_{d} / F_{c}$, where $w$ is the weight of material above the intruder after the penetration process, $w_{T}$ is the total weight of the sample, $F_{d}$ is the force applied to push the disk down and $F_{c}$ is the characteristic value used for the cohesion force. As depicted, the force to pull up the intruder disk can indeed be as much as three times higher than the force to push it down. Note that the fraction of active bonds in the final packing strongly depends on the "penetration strength". For low values of $F_{d} / F_{c}$, where the intruder is not pushed into the structure very deeply, most of the bonds are still active. For $F_{d} / F_{c} \sim 0.33$, e.g., $93 \%$ of the bonds are active with respect to the final structure, corresponding to $97 \%$ of the original bonds of the initial structure, i.e. very few bonds are broken. Also, for $F_{d} / F_{c} \sim 1$, where the intruder almost reaches the bottom, still $68 \%$ of the bonds are active ( $78 \%$ of the original bonds). As the intruder can only go down until reaching the bottom, increasing the "penetration strength" further only leads to the breaking of more cohesive bonds. This

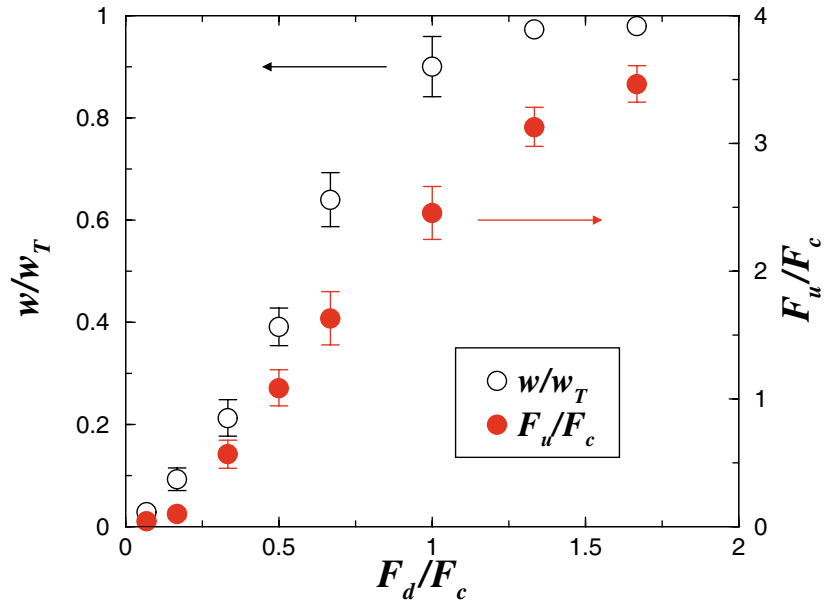

Fig. 2. Dependence of the normalized weight of material above the intruder after penetration $w / w_{T}$ (open circles, left ordinate) on the applied pushing force $F_{d} / F_{c}$ (normalized by cohesive force). In the same plot, we also show how the normalized force necessary to pull out the intruder $F_{u} / F_{c}$ (filled circles, right ordinate) varies with $F_{d} / F_{c}$.

indicates a stronger collapse of the structures which explains why the force to pull up the intruder still increases for high values of $F_{d} / F_{c}$.

The results presented above illustrate the typical behavior of such a fragile structure for a given initial density (here the initial volume fraction is about 0.43). Changing the volume fraction (density), the curves are shifted to the right for higher densities or to the left for lower densities, still showing the same qualitative behavior. We expect three-dimensional simulations to display a qualitative similar penetration behavior, but expect the intruder to experience less resistance of the material due to the additional degree of freedom. This effect has been found when comparing the pore stabilization mechanisms of two-dimensional and three-dimensional simulations $[9$, 15]. Disregarding the interstitial fluid motion keeps the model as simple as possible and nevertheless able to reproduce the main experimental observations. Of course the details of the collapse of the material may be influenced by the flow field of the surrounding fluid (e.g. [14,13]). We tested the influence of the fluid by introducing a viscous drag on the grains. For this, the drag coefficient of water and a typical grain size of $d=344 \mu \mathrm{m}$ have been used. The simulation results showed no significant difference.

The results of this section are also very important for the experiments presented in sect. 3.3 using a vane rheometer pushed into the investigated soil. When pushed in smoothly the thin vane blades will not damage too much the structure for the material to be considered unperturbed. This is one reason for choosing a relatively small intruder, being only three times larger than the grains, and being in the range or even smaller than the typical pore size of the structure. Additionally, this saves a lot of computation time as for larger intruders also the system sizes have to be larger. Preliminary studies with larger intruders show similar qualitative behavior and will be the subject of future studies. 


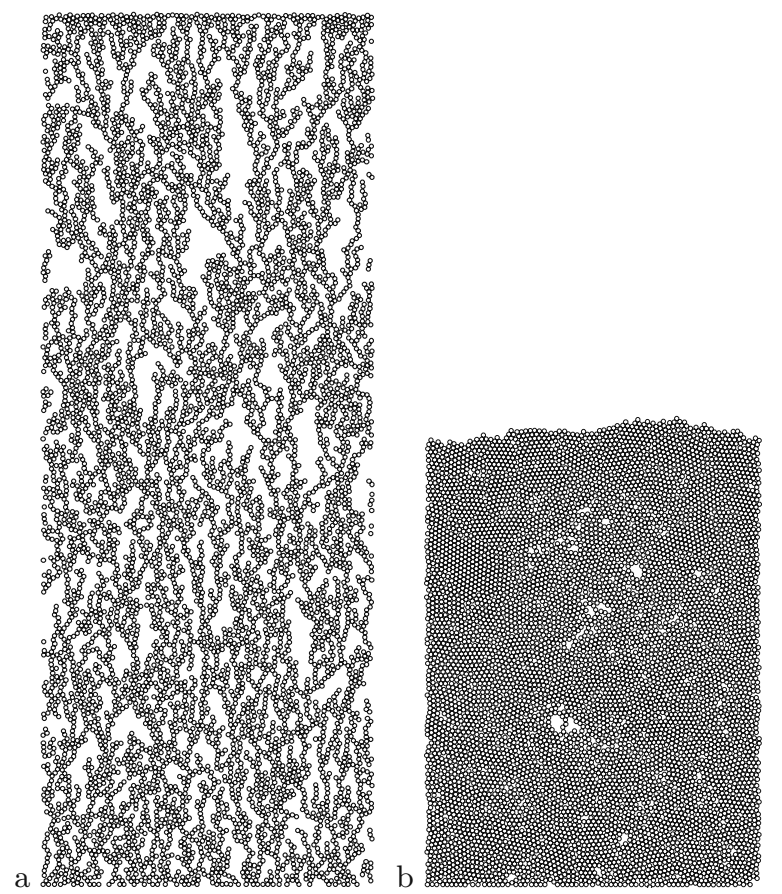

Fig. 3. Typical realizations of the granular system prepared for computational simulations of shear strength (periodic boundaries in horizontal direction). In (a) we show the (unperturbed) granular network of cohesive disks $\left(F_{c} / F_{g}=10^{4}\right)$ generated by ballistic deposition and contact dynamics, and in (b) the (perturbed) compact structure obtained after the collapse of the system due to a piston-like pushing force applied at the top. The system size is $51 \times 199$ in units of grain diameters, corresponding to $1.7 \mathrm{~cm} \times 6.7 \mathrm{~cm}$ when using the typical diameter $d=344 \mu \mathrm{m}$ of the grains in the experiments; b) $51 \times 106$.

\subsection{Shear resistance}

The unperturbed material is modeled by a ballistic deposition of particles driven by gravity, as an example of a process that generates very fragile structures. During this relatively slow process, bonds are allowed to form when particles stick to each other. As previously discussed, the origin of the attractive force can be explained in terms of cohesive bonds mediated by the bacteria living in the suspension [16]. For the bonds, we use the same model as described before, i.e. the bond strength is $F_{c}$, which is the dominant parameter determining the density of the system. The particles are sequentially deposited with a fixed time interval between each one to allow for relaxation. To get a very loose and fragile structure, an extremely large time between two depositions should be used. The procedure adopted here is to gradually increase the time interval. We only stop when the packing generated with the highest time interval has, within a given tolerance, the same particle density as the previous one. We show in fig. 3a that this procedure results in a highly porous, and therefore tenuously connected, network of grains. In practice, for the simulations presented here, depositing one particle each time step turned out to be already adiabatic, i.e. the density did not change any more (less than $0.1 \%$ ). Note that the generation process of the fragile structure used in our simulations somehow resembles the processes of formation of natural collapsing suspensions/soils investigated in in situ experiments [16]. This will later be important to be able to compare the experiments with our model (sect. 3.3).

With the purpose to simulate the shear strength after the collapse, we implement in our model a pistonlike pushing force acting on the particles at the top. In this situation, the collapse of the granular network that starts from the upper region of the system is analogous to a gradual increase in the effective piston weight, leading to an acceleration of the compaction mechanism. During this process bonds will break without reforming again up to the point in which most of the bonds are broken (see fig. $3 \mathrm{~b}$ ). This behavior will be valid for all materials where the time scale of bond formation is larger than that of the collapsing and measurement, or where the bonds are not able to reform again at all, as e.g. in the in situ experiments (sect. 3.3). After the collapse only $8.5 \%$ of the bonds are active (i.e. cohesive contacts) corresponding to about $13 \%$ of the active bonds of the initial structure, i.e. most of the bonds are broken. As the number of contacts increases during the collapse the coordination number increases from slightly above $2(2.05)$ to about 3.17 , whereas the volume fraction increases from about 0.4 to about 0.77 . The value for the coordination number is almost the same as within the channel after the compaction by an intruder (cf. sect. 3.1: coordination number about 3.2), whereas the fraction of active bonds, although very low, is still higher than in the intruder case. This can be understood because the intruder destroys the bonds very efficiently on its way down. Then, the material settles again without almost no active bonds in the "channel". In the case of compacting the whole system "externally" as described above, not all bonds have to be broken to compact the system. For the case of very large intruders one expects both situations: One part of the system below the intruder is compacted similar to the whole system in fig. 3b. Another part of the system collapses over the intruder within its "channel".

We calculate the shear strength of the unperturbed and perturbed structure as follows. At a given depth, we apply a constant force in the horizontal direction to a randomly chosen particle and observe how far it can move in this direction. Our assumption here is based on the fact that in real experiments the rheometer creates a thin shear layer at the upper and lower edges of the vane. This layer is represented in our model by these sample particles subjected to a constant force. By changing the force using nested intervals, one can calculate within a given numerical tolerance the minimum force necessary to move this particle at a distance that is sufficiently large to sample the disordered porous geometry (e.g., approximately 20 particle diameters away). This procedure is then repeated for different particles at the same depth to produce an average shear strength value. During this procedure, measuring the threshold in a static system when motion sets in, it is justified to disregard the fluid motion.

The results in fig. 4 show the variation with depth of the shear strength averaged over 10 different particles 


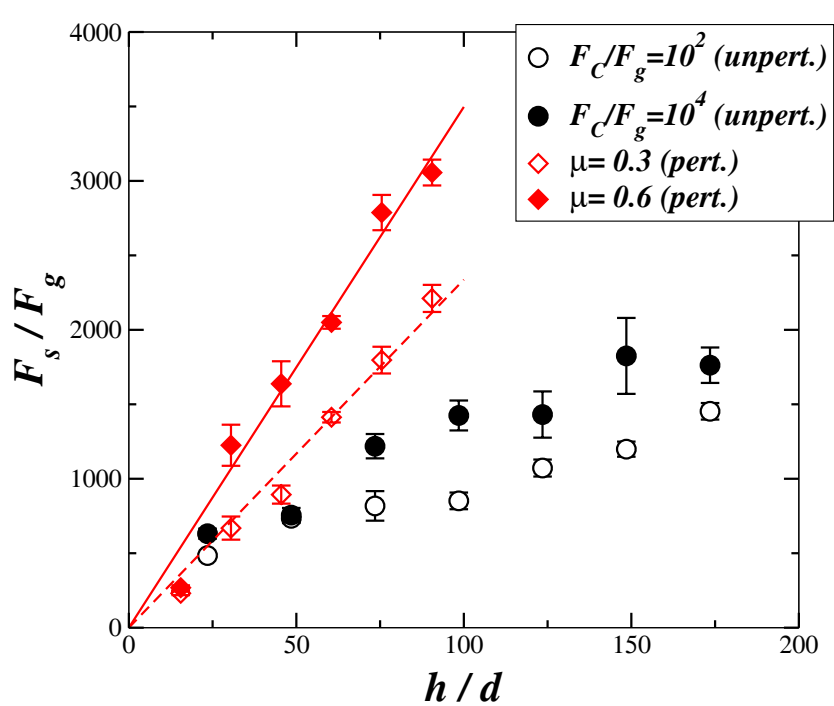

Fig. 4. Dependence of the simulated shear strength $F_{s}$ (normalized by the gravitional force $F_{g}$ of each grain) on the depth for unperturbed and perturbed systems (depth $h$ in units of grain diameters $d$ ). Compared to the perturbed system (diamonds), the unperturbed grain structure shows only a weaker dependence on the depth. This is similar to the behavior of a real collapsing suspension/soil (see fig. 5). For the unperturbed system, an increase by a factor of 100 in the cohesion force does not change substantially the behavior of the shear strength. For the perturbed system we also show two different values of the friction coefficient $\mu$ (0.3 and 0.6). A higher friction coefficient leads to a stronger increasing shear strength as indicated by the slopes $(a \approx 23.4$ for $\mu=0.3$ and $a \approx 35$ for $\mu=0.6)$ of the linear fit as in eq. (1).

for both unperturbed and perturbed systems. The cohesion forces are dominant in the unperturbed state while the contribution of friction forces governs the dynamical behavior of the perturbed material. The model results obtained with the unperturbed system show only a minor dependence of the shear strength on the depth compared to the perturbed structure. Furthermore, for the unperturbed system the shear strength does not extrapolate to zero in the limit of low depths. Additionally, we observe that a significant increase in the cohesion force intensity (e.g., by a factor of one hundred) does not lead to a substantially different behavior of the shear strength. This is a consequence of two distinct effects, namely that i) the particle density decreases with the cohesion force, and that ii) a larger shear strength is necessary to move a particle in a more compact medium (i.e., a high-density packing with volume fraction of about 0.69 and coordination number about 3). As also shown in fig. 4, the shear strength increases linearly with depth for the case of the perturbed material. Expectedly, as we observe in the same plot, an increase in the friction coefficient generally increases the average shear strength. Using the slopes of fig. 4, one can for the perturbed case calculate the effective friction coefficient of the material by simply deviding the shear strength at a certain depth by the weight of the particles above that depth. Here we use the full depth of the whole structure to achieve highest accuracy. For our simulation this results in an effective friction coefficient of $0.32(\mu=0.3)$ and 0.48 $(\mu=0.6)$. The relatively high values and in particular the large difference cannot solely be explained by the presence of friction. It can better be understood by the fact that there are still active bonds present in the structure, enhancing the strength of the material.

\subsection{Comparison to experiments}

In order to test our physical model, we use experimental data from investigations of a specific type of a natural collapsing suspension/soil [16]. This comparison between the real data and our contact dynamics model can only be made qualitatively due to obvious difficulties in obtaining reasonably precise experimental measurement of any in situ microscopic parameters.

The experimental data come from a natural reserve called Lençois Maranhenses located in the North-East of Brazil [16]. We found that at the shore of many drying lakes in this place, it is common to find a special type of a collapsing suspension/soil consisting of an impermeable crust lying above a metastable suspension of grains [17]. The slow drying process of the lakes, including continuous deposition of grains transported by the wind from the adjacant dunes into the lakes, is consistent with the slow limit of depositing grains used in the simulations (sect. 3.2) leading to very fragile structures. Provided one does not exert on the surface a pressure higher than $p_{c}=10-20 \mathrm{kPa}$, it is possible to step on it and the surface will elastically deform in a very similar way to what happens when one walks on a waterbed. These deformations visibly extend over a couple of meters. If the pressure $p_{c}$ is exceeded, the surface cracks in a brittle way producing a network of tensile cracks [16]. After that we observe the separation of the excess pore-water from a repacked and wet sand soil [18] with pronounced shear thinning behavior [19]. Because the collapse of this suspension/soil is irreversible, we had to study its rheology and strength in situ. This irreversible collapse leading to a compacted material with reduced pore volume could be qualitatively reproduced in our simulations, as presented before (cf. sects. 3.1 and 3.2).

By placing light plates on the surface, we could walk on the suspension/soil without visually modifying it and make various measurements before and after the collapse. The most striking result concerns the shear strength $\tau$ measured with a vane rheometer. The vane consists of 4 thin blades (see inset in fig. 5). During the measurement the vane rotates cutting out a cylindrical area of the material. In this respect the results of sect. 3.1 are very important, as the rheometer has to be pushed into the investigated soil, before measuring. The thin vane blades ensure that when pushed in smoothly enough most of the structure in the rotated cylinder is not destroyed by the vane. Thus, we were really able to measure the properties of the unperturbed material.

The vane rheometer measures both the threshold stress and the steady-state stress. Here we measure the maximal 


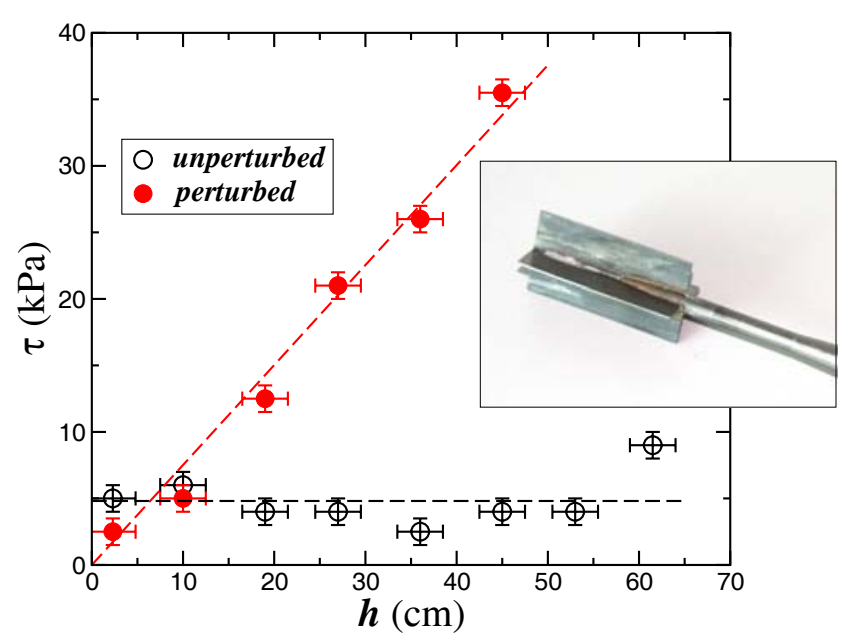

Fig. 5. Experimental measurements of the shear strength as a function of depth before (open circles) and after (filled circles) the collapse of the suspension/soil. For the measurements, we used a vane rheometer Geonor H-60 shown in the inset. The vertical error bars indicate the read-off accuracy of the vane rheometer, the horizontal error bars are given by the height of the vane. The least-squares fit to data of a linear function $\tau=a h$ gives $a=0.75 \pm 0.03 \mathrm{kPa} / \mathrm{cm}$ after the collapse. The shear strength of the unperturbed suspension/soil (before the collapse) follows an approximately constant behavior $\tau \approx 5 \mathrm{kPa}$ until reaching the bottom of the system, which was at about $60 \mathrm{~cm}$ below the surface for this case.

torque necessary to rotate the vane and, knowing its geometry, it is possible to calculate the threshold stress below which the vane does not move [20]. As shown in fig. 5, before destroying the structure, $\tau$ is essentially constant up to the bottom of the basin and then it rapidly increases. In order to reproduce this behavior with our model, simulations with much larger system sizes would be necessary. After the system collapsed and the water came out, $\tau$ linearly increases with depth $h$

$$
\tau(h)=a h,
$$

with $a=0.75 \pm 0.03 \mathrm{kPa} / \mathrm{cm}$. We conclude from our measurements that the soil essentially consists of a suspension with depth-independent shear strength. After the collapse, it becomes a soil dominated by the Mohr-Coulomb friction criterion for its shear strength. Such a behavior is again consistent with our model simulations of a collapsing soil/suspension. Summarizing, we found a cross-over from a yield stress material with a threshold value independent of depth to a Coulomb material after the collapse. At least, in the experiments the depth-independent threshold value can be clearly seen (fig. 5).

The analysis of this collapsing soil shows grains with a typical size of $d=344 \mu \mathrm{m}$ and standard deviation $\sigma_{d}=120 \mu \mathrm{m}$ as well as the presence of a huge amount of cyanobacteria and diatomacea of various types [16]. During the drying of the lakes, these organisms are responsible for the formation of the elastic and impermeable crust which prevents further water from evaporating out of the metastable suspension of grains. As reported in previous studies [17], we confirmed that the cohesion force between grains inside the suspension is mediated by the cyanobacteria present in the system.

\section{Conclusion}

Our model is capable to simulate the most important features of a real collapsing suspension/soil. Most features are important for many types of collapsing suspension/soil including wet and dry quicksand, and the model may also be applied for the collapse of cohesive powders or snow. Shed by bacteria in a highly unstable granular skeleton, this suspension/soil can catastrophically collapse. During this rapid segregation, objects lighter than water, once pushed deep down through the collapsing suspension, can be irreversibly trapped under the debris of disassembled grains and clusters of grains. According to our simulation results, this behavior appears to be a general feature of a collapsing suspension/soil. In our case, the fraction of still active bonds with respect to the total number of contacts is a very useful number for describing the collapsed structures microscopically. Moreover, our model results show similar behavior for the shear strength of the real material in both unperturbed and perturbed phases, showing a cross-over between a yield stress material to a Coulomb material after the collapse. As a challenge for future work, we intend to investigate the effect of size and shape of the intruder on the "drag" force exerted by the granular network [21] in the presence of cohesive forces.

This research was supported by a grant from the G.I.F., the German-Israel Foundation for Scientific Research and Development. Furthermore, we thank CNPq, CAPES, FUNCAP, FINEP, the Volkswagenstiftung and the Max Planck Prize for financial support.

\section{References}

1. J.K. Mitchell, K. Soga, Fundamentals of Soil Behavior, 3rd edition (Wiley, Hoboken, New Jersey, 2005).

2. Y.M. Reznik, Eng. Geol. 78, 95 (2005).

3. T. Ayadat, A. Hanna, Geotech. Test. J. 30, 312 (2007).

4. S. Manley et al., Phys. Rev. Lett. 94, 218302 (2005).

5. J. Heierli, J. Geophys. Res. (Earth Surf.) 110, F02008 (2005).

6. M. Jean, J.J. Moreau, Contact Mechanics International Symposium (Presses Polytechniques et Universitaires Romandes, Lausanne, 1992), pp. 31-48; J.J. Moreau, Eur. J. Mech. A-Solid 13, 93 (1994).

7. T. Unger, J. Kertesz, Modelling of Complex Systems, Seventh Granada Lectures 2003 (Melville, New York, AIP, 2003), pp. 116-138, cond-mat/0211696.

8. L. Brendel et al., The Physics of Granular Media (WileyVCH, Berlin, 2004), pp. 325-340.

9. D. Kadau et al., Phase Trans. 76, 315 (2003).

10. A. Taboada et al., Phys. Rev. Lett. 97, 098302 (2006).

11. Y. Noh, Fluid Dyn. Res. 27, 129 (2000).

12. D. Lohse et al., Nature 432, 689 (2004). 
13. J.R. Royer et al., Nature Phys. 1, 164 (2005).

14. G. Caballero et al., Phys. Rev. Lett. 99, 018001 (2007).

15. G. Bartels et al., Granular Matter 7, 139 (2005).

16. D. Kadau et al., Granular Matter 11, 67 (2009).

17. A. Danin, Flora 167, 409 (1978); J. Arid Environ. 21, 193 (1991).
18. W.R. Parker, Nature 210, 1247 (1966).

19. A. Khaldoun et al., Nature 437, 635 (2005).

20. C.R.I. Clayton et al., Site Investigation (Blackwell Science, Oxford, 1995).

21. R. Albert et al., Phys. Rev. Lett. 82, 205 (1999). 